ARTICLE

\title{
Intertwined density waves in a metallic nickelate
}

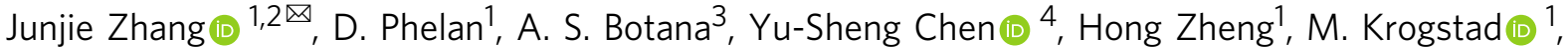 \\ Suyin Grass Wang (1) 4, Yiming Qiu (1) 5, J. A. Rodriguez-Rivera (1) 5,6, R. Osborn (1) 1, S. Rosenkranz (1) 1, \\ M. R. Norman ${ }^{1} \&$ J. F. Mitchell ${ }^{1 凶}$
}

Nickelates are a rich class of materials, ranging from insulating magnets to superconductors. But for stoichiometric materials, insulating behavior is the norm, as for most late transition metal oxides. Notable exceptions are the 3D perovskite $\mathrm{LaNiO}_{3}$, an unconventional paramagnetic metal, and the layered Ruddlesden-Popper phases $\mathrm{R}_{4} \mathrm{Ni}_{3} \mathrm{O}_{10}$, $(\mathrm{R}=\mathrm{La}, \mathrm{Pr}, \mathrm{Nd})$. The latter are particularly intriguing because they exhibit an unusual metal-to-metal transition. Here, we demonstrate that this transition results from an incommensurate density wave with both charge and magnetic character that lies closer in its behavior to the metallic density wave seen in chromium metal than the insulating stripes typically found in single-layer nickelates like $\mathrm{La}_{2-x} \mathrm{Sr}_{x} \mathrm{NiO}_{4}$. We identify these intertwined density waves as being Fermi surface-driven, revealing a novel ordering mechanism in this nickelate that reflects a coupling among charge, spin, and lattice degrees of freedom that differs not only from the single-layer materials, but from the 3D perovskites as well.

\footnotetext{
${ }^{1}$ Materials Science Division, Argonne National Laboratory, Lemont, IL 60439, United States. ${ }^{2}$ Institute of Crystal Materials, State Key Laboratory of Crystal Materials, Shandong University, 250100 Jinan, Shandong, China. ${ }^{3}$ Department of Physics, Arizona State University, Tempe, AZ 85287, United States. ${ }^{4}$ ChemMatCARS, The University of Chicago, Lemont, IL 60439, United States. ${ }^{5}$ NIST Center for Neutron Research, National Institute of Standards and Technology, Gaithersburg, MD 20899, United States. ${ }^{6}$ Department of Materials Science, University of Maryland, College Park, MD 20742, United States.

凶email: junjie@sdu.edu.cn; mitchell@anl.gov
} 
T he central challenge in harnessing the unrivaled diversity of transition metal oxides (TMO) is understanding (and manipulating) how charge and spin influence their properties. Gaining such understanding and control is made difficult by the many highly correlated degrees of freedom ubiquitously present in TMO. As a case in point, the family of rare earth nickel oxide perovskites, $\mathrm{RNiO}_{3}(\mathrm{R}=\mathrm{La}, \mathrm{Pr}-\mathrm{Lu})$, presents an important archetype TMO for investigating the nexus between charge localization and itineracy and their correlation with magnetism. These well-studied $\mathrm{Ni}^{3+}\left(\mathrm{d}^{7}\right)$ perovskites exhibit a commensurate antiferromagnetic insulating ground state for all $\mathrm{R}$ except La, and the ordering wavevectors $\left(\mathbf{q}_{\mathrm{MIT}}\right)$ driving the metal-insulator transition (MIT) and magnetic ordering are commensurate with respect to the parent cubic perovskite ${ }^{1}$. On the other hand, $\mathrm{LaNiO}_{3}$ is a good metal that remains rhombohedral at all temperatures. It shows no intrinsic, long-range ordered antiferromagnetism in bulk samples ${ }^{2-5}$, although evidence of short-range bond disproportionation associated with $\mathbf{q}_{\text {MIT }}$ has been reported ${ }^{6}$. By reducing the dimensionality, insulating ground states with commensurate stripe or checkerboard charge and spin-ordering are found in Sr-doped $\mathrm{La}_{2} \mathrm{NiO}_{4}$ (LSNO), the $n=1$ member the quasi-2D Ruddlesden-Popper (R-P) series $\mathrm{La}_{n+1} \mathrm{Ni}_{n} \mathrm{O}_{3 n+1}$, throughout its doping range between $\mathrm{Ni}^{2+}\left(\mathrm{d}^{8}\right)$ and $\mathrm{Ni}^{3+}\left(\mathrm{d}^{7}\right)^{7}$. Incommensurate charge and spin modulations observed in LSNO have been shown to arise from microscopic mixtures of commensurate stripe phases ${ }^{8}$. Commensurate charge-stripes and spin-stripes have also been identified recently in another low-dimensional insulating nickelate, $\mathrm{La}_{4} \mathrm{Ni}_{3} \mathrm{O}_{8}$, also at $1 / 3$-hole doping, albeit here $\mathrm{Ni}^{1.33+}$ $\left(d^{8.67}\right)^{9,10}$. Collectively through these systems, our current understanding of the rare earth nickelates has been framed as lying poised between localized and itinerant behavior and between magnetic order and disorder with both sectors governed by real-space charge and spin interactions.

Here, we report a dramatically different behavior in a higher order $(n=3) \mathrm{R}-\mathrm{P}$ nickelate, $\mathrm{R}_{4} \mathrm{Ni}_{3} \mathrm{O}_{10}(\mathrm{R}=\mathrm{La}, \mathrm{Pr}, \mathrm{Nd})$, in which we find intertwined charge-order and spin-order developing at a metal-to-metal transition (MMT) whose precise mechanism has remained an open question for nearly 25 years ${ }^{11-18}$. Combining single crystal synchrotron $\mathrm{x}$-ray and neutron diffraction, we find charge and spin-superlattice (SL) reflections below the MMT with incommensurate propagation vectors $\mathbf{q}_{\mathrm{c}}=\left(0, q_{c}, 0\right)$ and $\mathbf{q}_{\mathrm{s}}=(0,1-$ $\left.q_{s}, 0\right)$, respectively, with $q_{c}=2 q_{s}$ as expected for a system with coupled charge and spin order. We present models for these intertwined density waves that reproduce the experimental observations semi-quantitatively. In the charge sector, our data are uniquely consistent with a CDW centered on the Ni sites, establishing that the low energy electronic degrees of freedom in this system lie on the transition metal rather than on oxygen. The lack of local moments on La unequivocally establishes that the SDW is associated with the $\mathrm{Ni}$ ions. A measured compositional dependence of the incommensurate $\mathbf{q}_{\mathrm{c}}$ combined with DFT calculations argue that these itinerant systems order via a Fermi surface nesting mechanism, in this way resembling elemental chromium ${ }^{19}$ more than nickel oxides. As such, the unexpected behavior found in $\mathrm{R}_{4} \mathrm{Ni}_{3} \mathrm{O}_{10}$ represents an important bridge between the paramagnetism and latent charge-ordering and spin-ordering of $3 \mathrm{D}$ metallic $\mathrm{LaNiO}_{3}$ at higher nickel valence and the polaronic behavior found in quasi-2D R $\mathrm{R}_{2-x} \mathrm{Sr}_{x} \mathrm{NiO}_{4}$ at lower nickel valence.

\section{Results}

Physical properties. Figure $1 \mathrm{a}-\mathrm{c}$ show in-plane resistivity, inplane magnetic susceptibility, and heat capacity measured on $\mathrm{La}_{4} \mathrm{Ni}_{3} \mathrm{O}_{10}$ single crystals, which adopt a pseudo-orthorhombic Bmab structure shown in Fig. 2a (properly indexed as monoclinic,

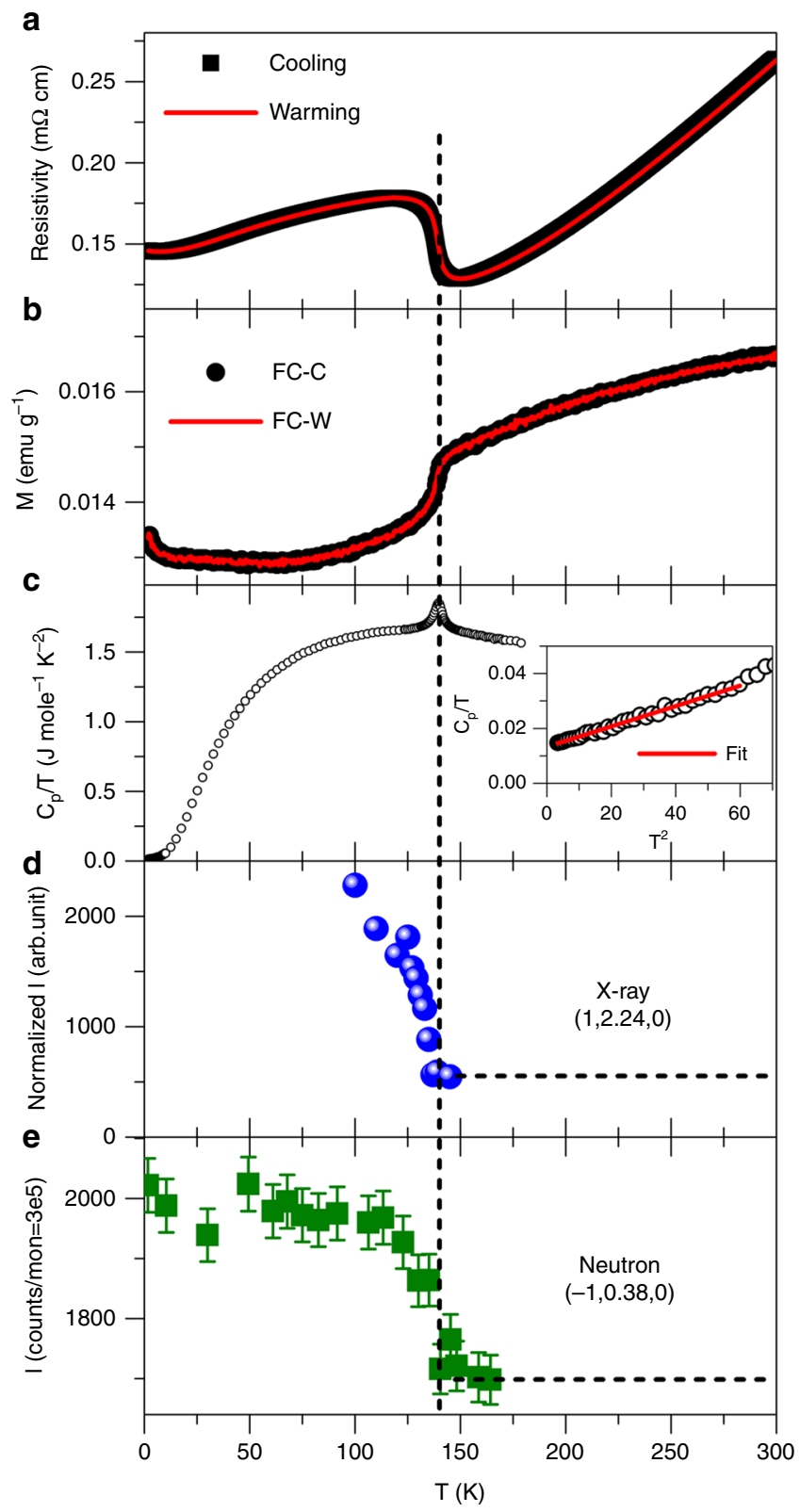

Fig. 1 Physical properties and order parameter of $\mathrm{La}_{\mathbf{4}} \mathrm{Ni}_{\mathbf{3}} \mathbf{O}_{\mathbf{1 0}}$. ab-plane (a), resistivity in zero field and (b), magnetic susceptibility measured under $0.4 \mathrm{~T}$ upon cooling and warming. c Heat capacity. Inset shows fit to low temperature data. $\mathbf{d}$ Temperature dependence of integrated intensity at $(1,2.24,0)$ measured with $x$-rays. e Temperature dependence of integrated intensity at $(-1,0.38,0)$ measured with neutrons. Error bars represent one standard deviation.

$P 2_{1} / a$, see ref. ${ }^{11}$ and Supplementary Note 1). The in-plane resistivity (Fig. 1a) drops with decreasing temperature, indicating metallic behavior, and an anomaly is observed at $T_{\mathrm{MMT}} \approx 140 \mathrm{~K}$. We note that the temperature-dependent transport behavior of $\mathrm{La}_{4} \mathrm{Ni}_{3} \mathrm{O}_{10}$ near $T_{\text {MMT }}$ resembles that reported for several density wave materials including chromium ${ }^{19}$, purple bronzes ${ }^{20}$, and rare-earth tritellurides ${ }^{21}$. The in-plane magnetic susceptibility (Fig. 1b) shows a sharp decrease at $T_{\mathrm{MMT}}$, but it does not follow either a Pauli or Curie-Weiss form above $T_{\mathrm{MMT}}$ in the temperature range measured, as it monotonically increases with increasing temperature. It does, however, resemble that of $\mathrm{CDW}$ materials such as the one-dimensional $\mathrm{K}_{0.3} \mathrm{MoO}_{3}{ }^{20}$, in which the temperature dependence of the susceptibility above the MMT was 
a

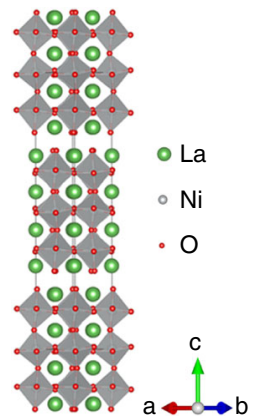

b

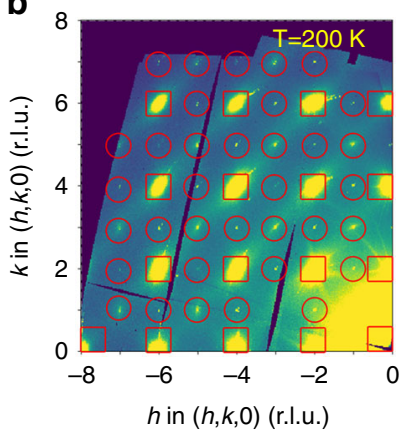

C

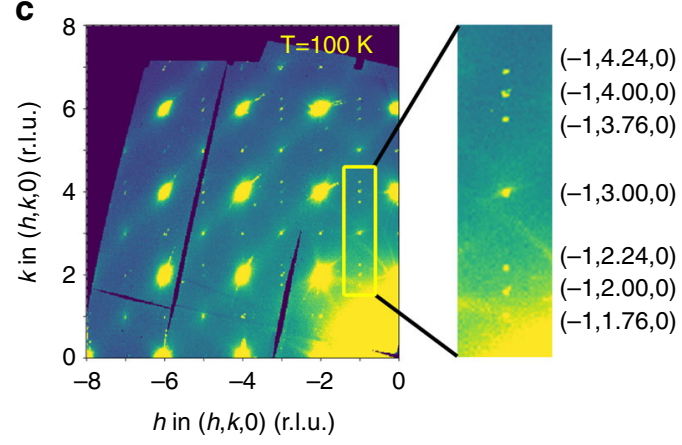

d

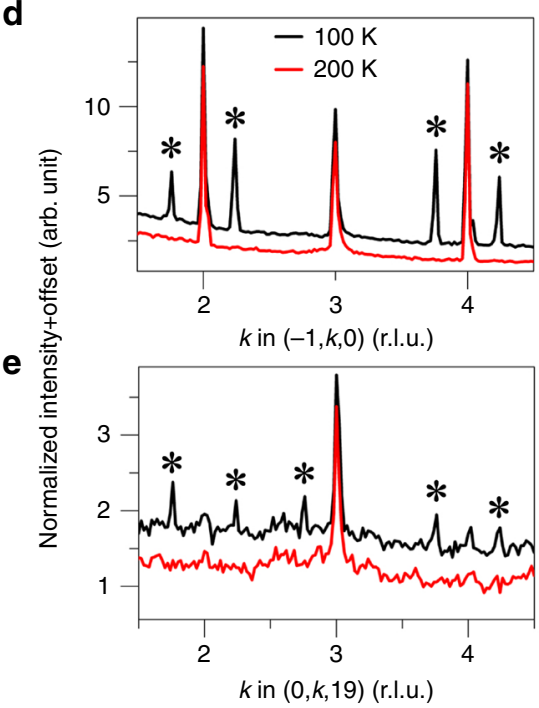

f

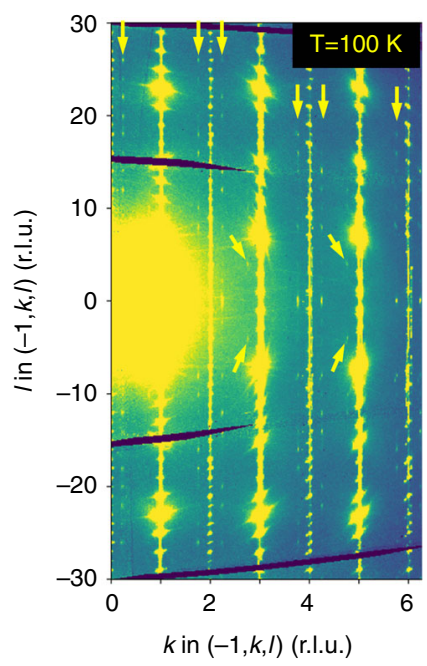

g

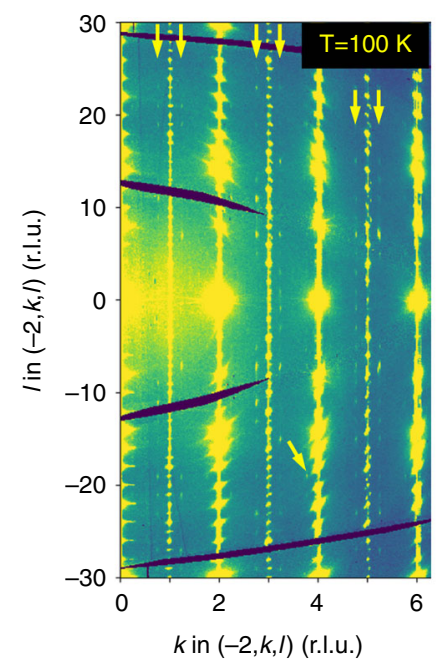

h

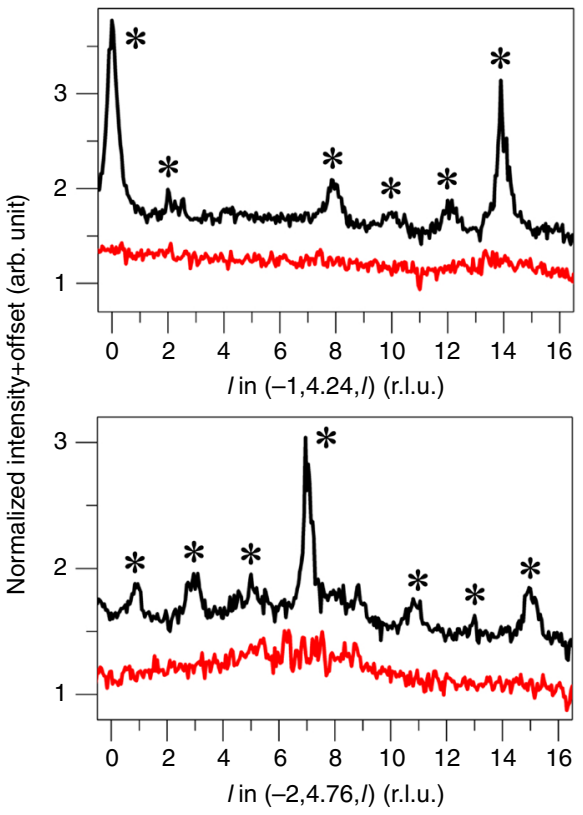

$\mathbf{i}$

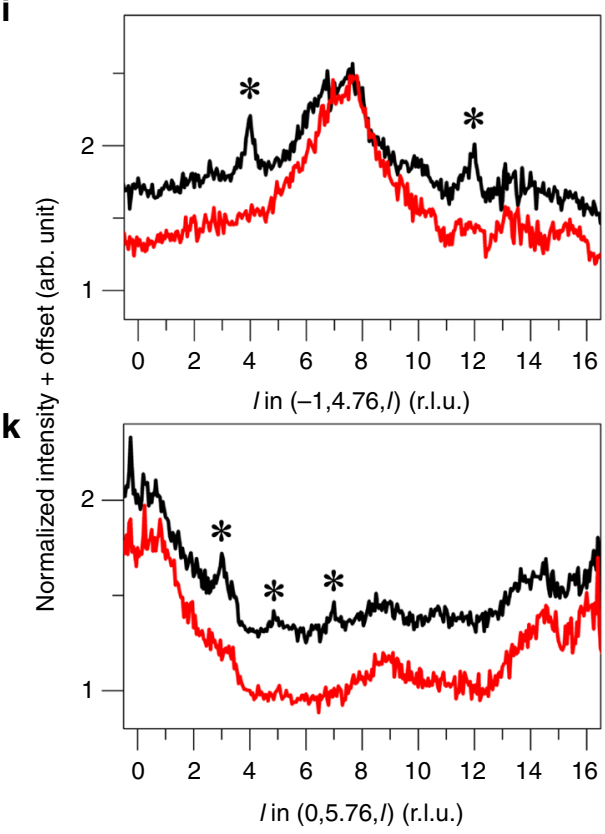

Fig. $\mathbf{2}$ Charge density wave order in $\mathrm{La}_{\mathbf{4}} \mathbf{N i}_{\mathbf{3}} \mathbf{O}_{\mathbf{1 0}}$. a Crystal structure of $\mathrm{La}_{4} \mathrm{Ni}_{3} \mathrm{O}_{10}$. The unit cell contains two trilayer perovskite-like blocks that are related through the $B$-centering operation of the Bmab space group. $\mathbf{b}$ hkO plane at $200 \mathrm{~K}$ as measured at Sector $15-\mathrm{ID}-\mathrm{D}$. The data are integrated over 0.05 r.l.u. in I. Hollow squares, fundamental Bragg peaks from high symmetry Bmab; hollow circles, Bragg peaks resulting from monoclinic distortion to $P 2_{1} / a$. c, $h k 0$ plane at $100 \mathrm{~K}$. d, e Line cuts through data along $k$ direction at 100 and $200 \mathrm{~K}$. The cuts have been integrated over 0.04 r.l.u. in $h$ and 0.05 r.l.u. in I. f, $\overline{1} \mathrm{kl}$ plane at $100 \mathrm{~K}$. The data are integrated over 0.02 r.l.u. in $h(h=-1) . \mathbf{g} 2 \mathrm{kl}$ plane at $100 \mathrm{~K}$. h-k Line cuts through data along I direction at 100 and $200 \mathrm{~K}$. Note red for $200 \mathrm{~K}$ and black for $100 \mathrm{~K}$. The cuts have been integrated over 0.02 r.l.u. in $h$ and 0.04 r.l.u. in $\mathrm{k}$. In $\mathbf{d}$, e, h-k, the data are shifted for clarity. Asterisks $\left({ }^{\star}\right)$ mark the superlattice reflections. Yellow arrows in $\mathbf{f}, \mathbf{g}$ point to superlattice reflections. 
attributed to CDW fluctuations and a pseudogap in the electronic density of states ${ }^{22}$. The heat capacity of $\mathrm{La}_{4} \mathrm{Ni}_{3} \mathrm{O}_{10}$ at low temperature (inset, Fig. 1c) was fit to the typical form, $C_{p} / T=\gamma+$ $\beta T^{2}$, where $\gamma$ is the Sommerfeld coefficient and the $\beta T^{2}$-term arises from phonons. The fit leads to $\gamma=13.3 \mathrm{~mJ} \mathrm{~mole}^{-1} \mathrm{~K}^{-2}$ and $\beta=0.37 \mathrm{~mJ}$ mole $^{-1} \mathrm{~K}^{-4}$, which agrees with a previous measurement of $C_{p}$ on polycrystalline samples by Wu et al. ${ }^{23}(\gamma=$ $13.5 \mathrm{~mJ} \mathrm{~mole}{ }^{-1} \mathrm{~K}^{-2}$ ) and by Kumar et al. ${ }^{13}\left(\gamma=15.5 \mathrm{~mJ} \mathrm{~mole}{ }^{-1}\right.$ $\left.\mathrm{K}^{-2}\right)$; however, we find a significantly larger Debye temperature $\left(\theta_{D}=450 \mathrm{~K}\right)$ than that reported by either group $\left(\theta_{D}=256 \mathrm{~K}^{23}\right.$, $384 \mathrm{~K}^{13}$, respectively). For comparison, reported values for single crystal $\mathrm{LaNiO}_{3}$ are $\gamma=11.7 \mathrm{~mJ}$ mole $\mathrm{K}^{-1}$ and $\theta_{D}=465 \mathrm{~K}^{2}$. From the Sommerfeld coefficient, $\gamma=\pi^{2} k_{B}{ }^{2} N\left(E_{F}\right) / 3$, the density of states at the Fermi level, $N\left(E_{F}\right)$, in $\mathrm{La}_{4} \mathrm{Ni}_{3} \mathrm{O}_{10}$ is estimated to be 1.9 states $\mathrm{eV}^{-1}$ per $\mathrm{Ni}$, significantly less than that of $\mathrm{LaNiO}_{3}$, 5.0 states $\mathrm{eV}^{-1}$ per Ni. Using the paramagnetic band structure, we find $N\left(E_{F}\right)=2.6$ states $\mathrm{eV}^{-1}$ per $\mathrm{Ni}$, which is substantially larger than the measured value. This suppression of states at $E_{F}$ further corroborates our inference of a pseudogap drawn from the magnetic susceptibility and transport data.

Charge density wave order. Using single crystal synchrotron $\mathrm{x}$ ray diffraction, we observed charge superlattice (SL) peaks in $\mathrm{La}_{4} \mathrm{Ni}_{3} \mathrm{O}_{10}$ below the MMT, suggesting the appearance of a charge-ordered state. The temperature dependence of the integrated intensity of one such SL reflection is shown in Fig. 1d. Its onset at $T_{\mathrm{MMT}}$ correlates with resistivity, magnetic susceptibility and heat capacity measurements. We note that these charge SL reflections have intensities typically $10^{4}$ times weaker than the strongest fundamental Bragg reflections, explaining why they have been unobserved in prior studies of polycrystalline samples.

Figure $2 \mathrm{~b}, \mathrm{c}$ show the $h k 0$ plane in reciprocal space at $200 \mathrm{~K}$ and $100 \mathrm{~K}$, respectively. At $200 \mathrm{~K}$, strong fundamentals appearing at integers $h k 0$ obey the Bmab reflection condition that they are both even, but additional scattering attributable to the $P 2_{1} / a$ cell is weakly observed as peaks that violate this rule. As shown in Fig. 2c, sharp first order superlattice reflections appear between certain fundamentals at $100 \mathrm{~K}$, located at $h=2 n+1$ and $k=2 m+$ $1 \pm q_{\mathrm{c}}$ ( $m, n$ integers). The appearance of SLs along $k$ coincides with the observation of a pronounced anomaly in the lattice parameter $b$ at the MMT $\mathrm{M}^{11,13}$. In this particular crystal, the SL reflections appear only along $k$, indicating a single domain modulation despite the near metrical equivalence of $a$ and $b$, which differ by $<1 \%$. We note that several other specimens that we investigated did show the presence of orthogonal modulations expected of a multidomain state.

Line cuts along $(-1, k, 0)$ and $(0, k, 19)$ are shown in Fig. $2 d$, e, respectively. By fitting the peaks, a modulation wavevector $\mathbf{q}_{\mathrm{c}}=$ $0.76 \mathbf{b}^{*}$ was obtained (see also Supplementary Figs. 1 and 2). The width of the SL peaks is somewhat broader than that of the corresponding fundamental peaks (Supplementary Fig. 3), indicating an in-plane correlation length, $\xi_{\mathrm{ab}} \sim 100 \AA$. This correlation length is comparable to that reported for stripe-order in $\mathrm{La}_{1.67} \mathrm{Sr}_{0.33} \mathrm{NiO}_{4}{ }^{24}$ and charge order in $\mathrm{YBa}_{2} \mathrm{Cu}_{3} \mathrm{O}_{6.67}{ }^{25}$.

Higher harmonics of the SL are not observed (for a discussion of sensitivity of our measurement to potential harmonics, see Supplementary Note 7 and Supplementary Fig. 12), demonstrating a sinusoidal character to the CDW. Furthermore, with a nominal doping level of $1 / 3$ in $\mathrm{La}_{4} \mathrm{Ni}_{3} \mathrm{O}_{10}$, the propagation vector would be expected at commensurate $\mathbf{q}_{c}$. These facts tend to rule out alternatives such as real-space charge stripes like those found in the 1/3-hole doped single layer R-P phase $\mathrm{La}_{1.67} \mathrm{Sr}_{0.33} \mathrm{NiO}_{4}{ }^{24}$ or square planar trilayer $\mathrm{La}_{4} \mathrm{Ni}_{3} \mathrm{O}_{8}$ (ref. ${ }^{9}$ ), subject to the caveat that a locally commensurate stripe phase with $q_{c}=2 / 3$ and a phase slip every $\approx 11$ diagonal rows cannot be ruled out ${ }^{26}$. We also point out that oxygen nonstoichiometry is unlikely to be the cause of the incommensurability. The oxygen content of La- and $\mathrm{Pr}_{4} \mathrm{Ni}_{3} \mathrm{O}_{10}$ are within 2 and 5 parts per thousand of 10.00 , respectively ${ }^{11}$, with La showing an oxygen deficiency and $\operatorname{Pr}$ an oxygen excess. Following the arguments of Yoshizawa et al. applied to $\mathrm{La}_{2-} \mathrm{Sr}_{x} \mathrm{NiO}_{2+\delta}$ (ref. ${ }^{8}$ ), an $\mathrm{O}$ deficiency of $\sim 0.08$ vacancies per formula unit, far in excess of what we measure in the case of La, would be needed to shift $q_{c}$ from $2 / 3$ to its observed value. Furthermore, as shown in Supplementary Fig. $2 b, q_{c}$ is consistent across three unique samples, an unlikely occurrence were oxygen stoichiometry controlling the incommensurability of the modulation.

Figure $2 \mathrm{f}$, g show $\overline{1} k l$ and $\overline{2} k l$ planes at $100 \mathrm{~K}$, and cuts along $l$ are shown in Fig. $2 \mathrm{~h}-\mathrm{k}$. SL peaks along $l$ occur at integers obeying the selection rule that $h+l$ is odd. This selection rule is shown in Fig. $2 \mathrm{~h}$, j. For $h=2 n+1$, SL peaks are located at $l=2$ $m$, while for $h=2 n$, SL peaks are observed at $l=2 m+1(m, n$ integers). In contrast, when $h$ and $l$ have the same parity, no SL reflections are observed above background. SL peaks in these figures are observed for $h, k$ of the same parity ( $k$ is defined by the integer from which $\mathbf{q}_{\mathrm{c}}=0.76 \mathbf{b}^{*}$ is measured). As shown in Fig. 2i, k, weaker SL peaks are also found for $h, k$ of mixed parity. Another notable feature is that the intensity in these cuts along $c^{*}$ is largest at $l=0$ and 14 for SL peaks associated with odd $h$ and odd $k$ fundamentals (Fig. $2 \mathrm{~h}$ ), but at $l=7$ for SL peaks at even $h$ and even $k$ (Fig. $2 \mathrm{j}$ ). The interval between maxima is similar to the ratio of the length of the $c$ axis to the $\mathrm{Ni}-\mathrm{O}$ layer separation within a trilayer $\left(\tau=c / d_{\mathrm{Ni}-\mathrm{Ni}}=7.2\right)$. The intensity distribution along $\mathbf{c}^{*}$ thus reflects a sinusoidal modulation of the trilayer unit structure factor with period $\tau$. A model of the CDW discussed below captures these features of the diffraction pattern. Finally, the width of SL peaks along $l$ is significantly broader than that of the nearby Bragg peaks, indicating a finite correlation length, $\xi_{c}$, of the charge order. Analysis of the peak width yields $\xi_{c} \approx 0.7 c \approx 21 \AA$ (Supplementary Fig. 4), verifying weak correlation of the charge order between neighboring unit cells along $c$ required to generate the selection rules discussed above.

Spin density wave order. Single crystal neutron diffraction data were measured to test for the presence of an SDW concomitant with the CDW. If such an SDW were present, a coupling between these two density waves is expected to occur such that $q_{c}=2 q_{s}{ }^{27}$. Figure 3a shows the $0 \mathrm{kl}$ plane in the reciprocal space of the neutron scattering data, with a background measured at $180 \mathrm{~K}$ subtracted (additional measurements in the $h k 0$ plane are discussed in the Supplementary Note 4 and Supplementary Fig. 5). SL reflections attributed to magnetic scattering are observed at $k=1 \pm q_{s},-1 \pm q_{s}$. A cut along $(0, k, 2)$ is presented in Fig. $3 \mathrm{~b}$, where magnetic SL reflections appear at $k=-1.38$ and -0.62 , i.e., $-1 \pm q_{s}$. Noting that $(0,1)$ corresponds to the " $(\pi, \pi)$ " antiferromagnetic wavevector that is frequently employed in the cuprate literature (i.e., with reference to an undistorted $3.8 \AA \times$ $3.8 \AA$ tetragonal sub-cell), the SDW propagation vector in $\mathrm{La}_{4} \mathrm{Ni}_{3} \mathrm{O}_{10}$ was determined to be $\mathbf{q}_{\mathrm{s}}=0.62 \mathbf{b}^{*}$, that is $\mathbf{q}_{\mathrm{s}}=\left(0,1-q_{s}\right.$, 0 ), with $q_{s}=0.38$. With this assignment of the magnetic SL, $q_{c}=$ $2 q_{s}$ (Fig. 3d), as expected for a coupling between the CDW and SDW. The cut along $\left(0,1-q_{s}, l\right)$ shown in Fig. $3 c$ evidences strong SL peaks along $\mathrm{c}^{*}$ at $l= \pm 2, \pm 6,-10$, i.e., $l=4 n+2$. This intensity distribution suggests antiferromagnetically coupled planes separated by $n c / 8$, with $n$ odd. This would occur if the inner planes were non-magnetic and the outer planes were $\pi$ out of phase. However, a closer inspection shows weaker intensity appearing at $l= \pm 4$ and -8 as well, reflecting that the outer plane spacing is in reality $c / 3.6$ instead of $c / 4$. 
a

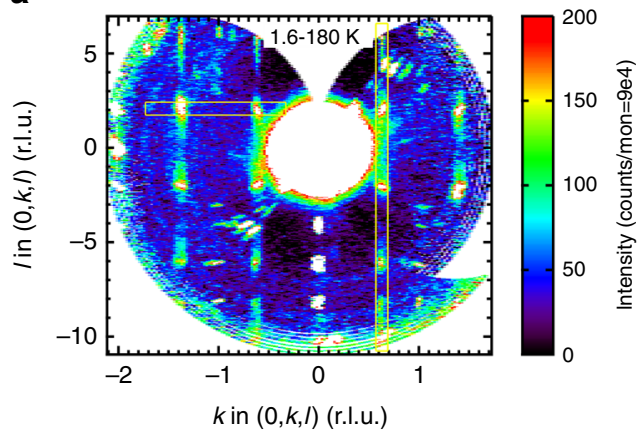

C

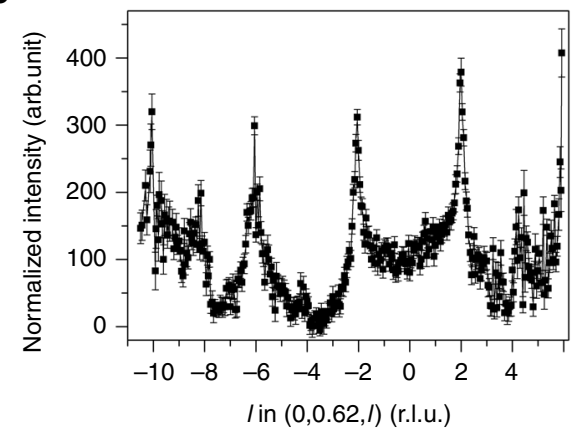

b

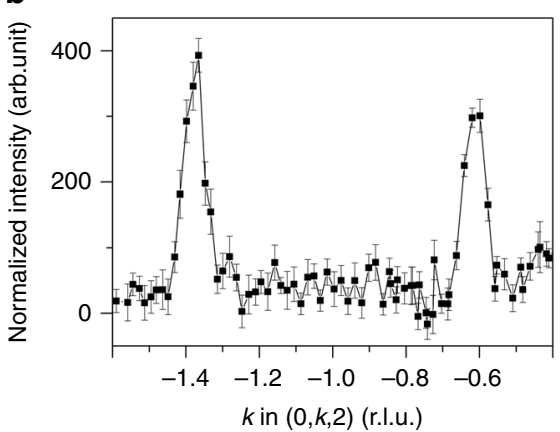

d

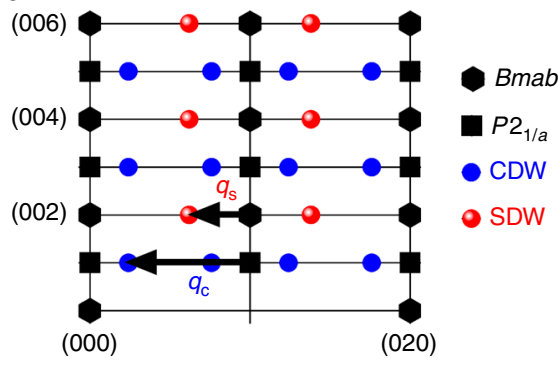

Fig. 3 Spin density wave order in $\mathbf{L a}_{\mathbf{4}} \mathbf{N i}_{\mathbf{3}} \mathbf{O}_{\mathbf{1 0}} . \mathbf{a}, 0 \mathrm{kl}$ plane with background $(180 \mathrm{~K})$ subtracted from $1.6 \mathrm{~K}$ data. $\mathbf{b}, \mathbf{c}$ Line cuts along $(0, k, 2)$ and $(0,0.62, I)$, respectively. $\mathbf{d}$ Schematic of the $\mathrm{Ok}$ plane showing location of $B m a b$ and $P 2 \sqrt{ } a$ fundamentals and both $C D W$ and SDW superlattice reflections. Error bars represent one standard deviation.

We show in Fig. 1e the temperature dependence of the integrated intensity measured at $(-1,0.38,0)$. As expected, the onset of intensity is observed at $T_{\mathrm{MMT}}$, coincident with the CDW. There is no compelling evidence in Fig. 1d, e for assigning charge or spin as the primary order parameter, and the onset temperature is the same for both within experimental resolution. In these ways, $\mathrm{La}_{4} \mathrm{Ni}_{3} \mathrm{O}_{10}$ behaves similarly to the related $\mathrm{La}_{4} \mathrm{Ni}_{3} \mathrm{O}_{8}$ system containing square planar $\mathrm{Ni}^{10}$.

Density wave models and simulations. We begin with the SDW. Prominent peaks at $l=2$ and 6 along the $(0,0.62, l)$ cut (Fig. $3 c$ ) indicate an approximate pattern of $l=4 n+2$ when $h=0$. This selection rule implies that the magnetic stacking pattern of the six planes in the unit cell (three per trilayer) is $\uparrow,-, \downarrow ; \uparrow,-, \downarrow$, where - represents a node. This argument holds regardless of the direction of spin polarization. Had the planes been spaced precisely $d=c / 8$ apart (as in the related compound $\mathrm{La}_{4} \mathrm{Ni}_{3} \mathrm{O}_{8}{ }^{10}$ ), then the $l=4 n+2$ selection rule would be exact. We note that such a magnetic ground state, with an SDW node on the inner planes, is unusual. A related example may be layered cuprates, where the inner and outer planes differ electronically due to different effective doping levels ${ }^{28}$. In $\mathrm{La}_{4} \mathrm{Ni}_{3} \mathrm{O}_{8}$, the magnetic stacking pattern is $\uparrow, \downarrow, \uparrow$ instead (leading to a prominent peak at $l=4$, which is notably absent here), with $\uparrow,-, \downarrow$ being an excited state.

The simplest model is to assume the in-plane behavior of the SDW has the form $\cos \left(\mathbf{q}_{\perp} \cdot \mathbf{r}_{\perp}\right)$ with $\mathbf{q}_{\perp}=\left(0,1-q_{s}\right)$ and $\mathbf{r}_{\perp}$ the inplane coordinates of the $\mathrm{Ni}$ ions. Our calculations implicitly assume that the spin direction is along a (i.e., in the basal plane and perpendicular to $\mathbf{q}_{\mathrm{s}}$ ), which is consistent with our experimental observations ${ }^{11}$, although additional (polarized) measurements would be required for absolute determination of the spin direction. For a given plane, the SDW corresponds to diagonal rows of aligned spins as found in $\mathrm{La}_{4} \mathrm{Ni}_{3} \mathrm{O}_{8}{ }^{10}$ and $\mathrm{La}_{1.67} \mathrm{Sr}_{0.33^{-}}$ $\mathrm{NiO}_{4}{ }^{27}$. We choose a commensurate value of $q_{s}=3 / 8$ to facilitate comparison to real space models. The resulting SDW pattern is shown schematically in Fig. 4b. The intensity distribution is calculated from

$$
I(\boldsymbol{k})=\left|\sum_{\mathbf{r}} \mathrm{c}_{\mathrm{z}} \mathrm{e}^{\mathrm{i} \mathbf{k} \cdot \mathbf{r}} \cos \left(\mathbf{q}_{\perp} \cdot \mathbf{r}_{\perp}\right)\right|^{2}
$$

with $c_{z}=1,0,-1 ; 1,0,-1$ encoding the magnetic stacking pattern along $c$ discussed above with the sum $\mathbf{r}$ over $\mathrm{Ni}$ atoms (normalized by their number). This expression accounts for the centering translation in the phasing between the trilayers. The resulting $(0, k, 2)$ and $(0,5 / 8, l)$ cuts are shown in Fig. $4 c, d$, respectively, and should be compared to the experimental data in Fig. 3b, c, respectively. In Fig. 4 d, note the small peak at $l=4$ and the larger peaks for even values of $l$ beyond $l=6$. This arises from the fact that the planes are spaced within the trilayer by $\sim c / 7.2$ rather than by $c / 8$. In Supplementary Fig. 7, results for different assumed SDW stackings along $c$ are also shown, along with a comparison of cuts along $l$ for $h=0$ and $h=-1$ in Supplementary Fig. 8.

We now turn to the CDW peaks. Strong CDW peaks are seen for $h+l=2 n+1$ and $k+l=2 n+1$. This pattern, as well as the presence of a strong peak at $l=7$ for certain momentum cuts like $(0,-3.24, l)$, implies that the CDW is (1) present on all planes, (2) is in-phase within a trilayer, and (3) is out-of-phase between successive trilayers. For (1), because $d \sim c / 7.2$ (explaining the strong peak at $l=7$ ) and because the pattern of CDW $l$ harmonics does not reflect that of the SDW discussed above, the CDW must be present on all planes. Condition (2) derives from the $B m a b(1 / 2,0,1 / 2)$ face-centered translation reflection condition, i.e., $h+l=2 n$. This general $B m a b$ condition becomes $h+l=2 n+1$ if the modulation in successive trilayers is $\pi$ out of 
a

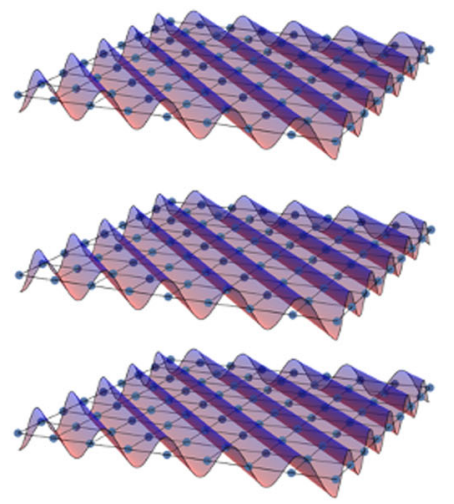

C

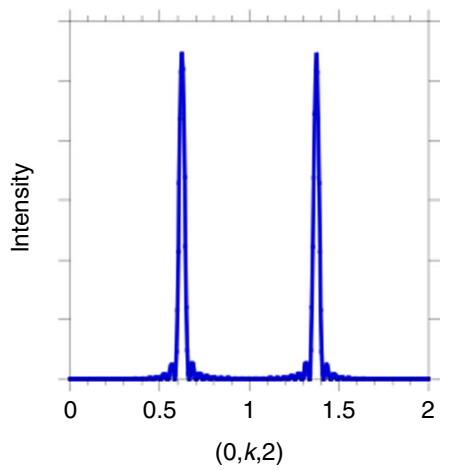

e

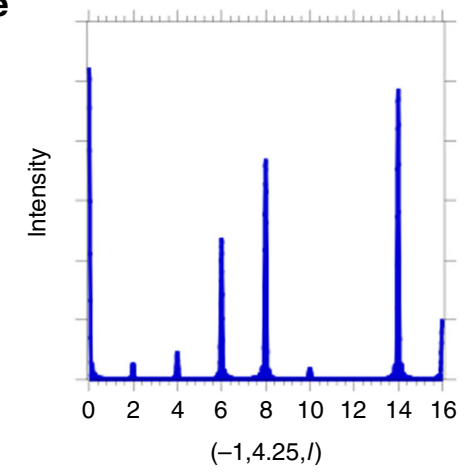

b

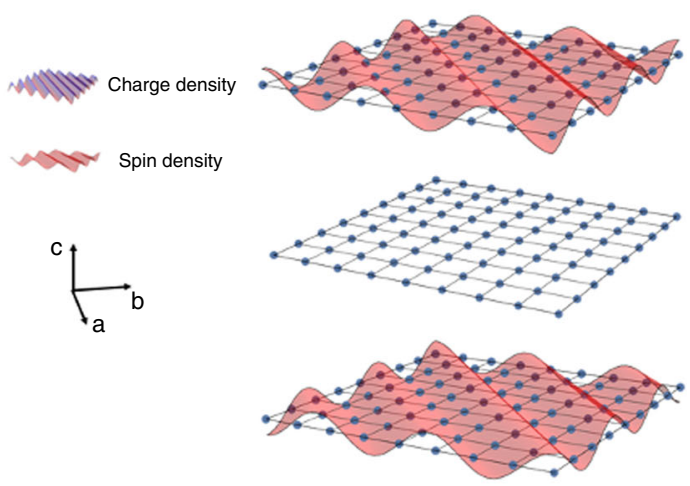

d

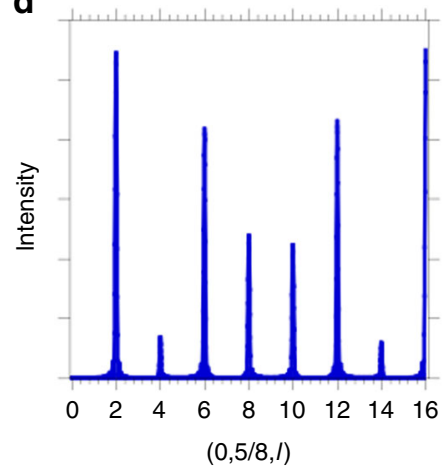

$\mathbf{f}$

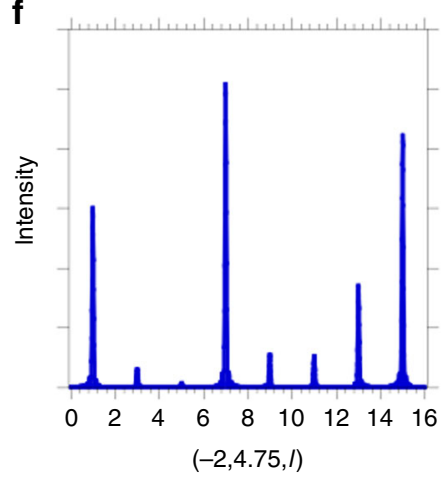

Fig. 4 Density wave simulations. a, b Model for the CDW and SDW plotted in the orthorhombic $a-b$ plane. One trilayer is shown. For $\mathbf{a}$, the CDW exists on all layers and is in-phase between the layers. For $\mathbf{b}$, the SDW has a node on the inner plane and is out-of-phase between the two outer planes. In addition, the CDW in $\mathbf{a}$ is out-of-phase between trilayers, but the SDW in $\mathbf{b}$ is in-phase. $\mathbf{c}, \mathbf{d}$ Predicted SDW diffraction intensities along $k$ and along $I$. e, $\mathbf{f}$ Predicted CDW diffraction intensities along $I$. e and $\mathbf{f}$ differ in having either even or odd $/$ peaks.

phase. The other condition, $k+l=2 n+1$, occurs because the $\mathrm{Ni}$ ion positions deviate only slightly from $(00 z)$. This slight deviation generates weak reflections that satisfy $k+l=2 \mathrm{n}$ as well. The resulting CDW pattern, modeled by $\boldsymbol{q}_{\perp}=\left(0,2-q_{c}\right)$ with $q_{c}=3 / 4$ (and $c_{z}=1,1,1 ;-1,-1,-1$ ), is shown in Fig. 4a. Representative cuts demonstrating strong CDW peaks are shown in Fig. 4 e, f. Note that $\mathbf{q}_{\mathrm{c}}=2 \mathbf{q}_{\mathrm{s}}$, as expected when relating the CDW peaks to those of the SDW. Results for different assumed CDW stackings along $c$, as well as contrasting different $k$ and $l$ cuts, are shown in Supplementary Figs. 9-11.

The fact that a simple cosine wave can explain the data, along with the observed incommensurability and the observation that this is a metal to metal transition, all support the picture of an itinerant density wave with a $2 k_{F}$ instability, as seen in chromium ${ }^{19}$. Unlike chromium, however, the CDW peaks in $\mathrm{La}_{4} \mathrm{Ni}_{3} \mathrm{O}_{10}$ are quite strong, and there is no evidence (Fig. 1d, e) that the CDW amplitude is secondary in the sense of the Landau theory of phase transitions (i.e., quadratic in the SDW amplitude). In this regard, $\mathrm{La}_{4} \mathrm{Ni}_{3} \mathrm{O}_{10}$ echoes its reduced analog, $\mathrm{La}_{4} \mathrm{Ni}_{3} \mathrm{O}_{8}$, though in that case one finds a semiconductor to insulator transition and a real space charge-stripe and spin-stripe scenario with a commensurate wavevector ${ }^{9,10}$. This behavior contrasts with chromium, where the CDW/strain wave is clearly a secondary order parameter ${ }^{29}$.

\section{Discussion}

Recent reports on the electronic structure of the related trilayer $\mathrm{R}$ $P$ phases $\mathrm{Pr}_{4} \mathrm{Ni}_{3} \mathrm{O}_{10}$ (ref. ${ }^{12}$ ) and $\mathrm{Nd}_{4} \mathrm{Ni}_{3} \mathrm{O}_{10}$ (ref. ${ }^{14}$ ) have stressed the importance of differentiating the inner layer and outer layers of the trilayer blocks. Indeed, because of symmetry, some degree of charge differentiation between the layers is inevitable. From a 
DFT perspective, only minor quantitative differences $(0.024$ electrons) are found between the inner and outer layers. The model presented above for the CDW posits a uniform charge density modulation in all three layers, but other models we have studied with nonuniform weights only differ in a quantitative sense (Supplementary Fig. 9). We note that differential doping could offer an explanation for the node in the SDW observed on the inner layer, as suggested for multi-layered cuprates ${ }^{28}$, though we remark that our modeling unambiguously finds that the CDW is present on the inner plane as well.

The effect of chemical or physical pressure on the MMT in the $\mathrm{R}_{4} \mathrm{Ni}_{3} \mathrm{O}_{10} \quad(\mathrm{R}=\mathrm{La}, \mathrm{Pr}, \mathrm{Nd})$ series has recently been shown through the systematic response of $T_{\mathrm{MMT}}$ to these parameters ${ }^{14,15}$. The monotonic variation of the transition is consistent with a Fermi surface driven instability sensitive to variables that can modify the electronic band structure and hence the relevant nesting vector $(\mathrm{s})^{30,31}$. Indeed, we find that by substituting $\mathrm{Pr}$ for La that the propagation vector responds to the effect of chemical pressure, albeit weakly, with $q_{c}=0.78$ for $\mathrm{Pr}_{4} \mathrm{Ni}_{3} \mathrm{O}_{10}$, and $q_{c}=2 q_{s}$ (Supplementary Fig. 6). In support of this nesting picture, we show calculations of the susceptibility from band theory in Supplementary Note 9 (Supplementary Fig. 13).

In some sense, one could regard $\mathrm{La}_{4} \mathrm{Ni}_{3} \mathrm{O}_{10}$ as a doped version of $\mathrm{LaNiO}_{3}$, which is a metal known to lie near an antiferromagnetic quantum critical point ${ }^{32}$. Indeed, $\mathrm{La}_{4} \mathrm{Ni}_{3} \mathrm{O}_{10}$, with an average $\mathrm{Ni}$ valence of $2.67\left(\mathrm{~d}^{7.33}\right)$, corresponds to $\mathrm{Ni}^{2+}$ and $\mathrm{Ni}^{3+}$ in a $1: 2$ ratio and as such is $1 / 3$ electron-doped relative to the parent $\mathrm{LaNiO}_{3}$ perovskite. With its trilayer structure, the ground state of quasi-2 $\mathrm{D} \mathrm{La}_{4} \mathrm{Ni}_{3} \mathrm{O}_{10}$ lies at a crossover between the paramagnetic 3D metal $\mathrm{LaNiO}_{3}$ and the insulating, more 2D single layer nickelates, $\mathrm{La}_{2-x} \mathrm{Sr}_{x} \mathrm{NiO}_{4}(x<1)$. Rather than the static real-space charge and spin stripes found in the latter materials when $x<0.5^{27}$, the result of this moderately strengthened coupling in $\mathrm{La}_{4} \mathrm{Ni}_{3} \mathrm{O}_{10}$ is an incommensurate charge-density and spin-density wave order that appears to be unprecedented in a $3 d$ oxide. Metallic conductivity coexisting with the CDW and SDW implies a partial gapping of the Fermi surface at $T_{\mathrm{MMT}}$. Indirect support for this latter point comes from the specific heat, where $N$ $\left(E_{F}\right)$ for metallic $\mathrm{LaNiO}_{3}$ is nearly three times larger than that of $\mathrm{La}_{4} \mathrm{Ni}_{3} \mathrm{O}_{10}$. Future studies using, for instance, high resolution ARPES and tunneling could shed light on this putative gapping (The gap reported in ref. ${ }^{16}$ results from orthorhombic backfolding of the $3 z^{2}-r^{2}$ bands, as can be seen near the $\Gamma$ point in Supplementary Fig. 13a. As such, it is unrelated to the CDW/ SDW gap discussed here).

We note that Jahn-Teller physics implied by the formal presence of $\mathrm{Ni}^{3+}$ is not important in $\mathrm{La}_{4} \mathrm{Ni}_{3} \mathrm{O}_{10}$, as evidenced through the suppressed orbital polarization between $x^{2}-y^{2}$ and $3 z^{2}-r^{2}$ states that we reported earlier from XAS measurements ${ }^{33}$. Indeed, this weak orbital polarization implies that bandwidth effects dominate over any potential Jahn-Teller orbital splitting in $\mathrm{La}_{4} \mathrm{Ni}_{3} \mathrm{O}_{10}$.

Finally, the relation of the incommensurate modulations observed here and the antiferromagnetic QCP identified for the perovskite $\mathrm{LaNiO}_{3}$ is not clear. This could be studied, for instance, by hole-doping $\mathrm{La}_{4} \mathrm{Ni}_{3} \mathrm{O}_{10}$ toward metallic $\mathrm{LaNiO}_{3}$. In a like vein, the relation of $\mathrm{La}_{4} \mathrm{Ni}_{3} \mathrm{O}_{10}$ to insulating $\mathrm{La}_{2-x} \mathrm{Sr}_{x} \mathrm{NiO}_{4}$ could be investigated by electron-doping instead, or else by studying the bilayer homolog $\mathrm{La}_{3} \mathrm{Ni}_{2} \mathrm{O}_{7}$. From such studies, it should be possible to build a unified understanding of this exceptional group of quantum materials.

\section{Methods}

Sample growth and characterization. Single crystals of $\mathrm{La}_{4} \mathrm{Ni}_{3} \mathrm{O}_{10}$ were grown using a vertical optical image high-pressure floating zone furnace at $20 \mathrm{bar} \mathrm{pO}_{2}$ (Model HKZ, SciDre, Germany (The identification of any commercial product or trade name does not imply endorsement or recommendation by the National Institute of Standards and Technology) $)^{11}$. Precursor powders of $\mathrm{La}_{4} \mathrm{Ni}_{3} \mathrm{O}_{10}$ were hydrostatically pressed into polycrystalline rods (length $=100 \mathrm{~mm}$, diameter $=$ $8 \mathrm{~mm}$ ) and sintered for $24 \mathrm{~h}$ at $1400^{\circ} \mathrm{C}$. $\mathrm{La}_{4} \mathrm{Ni}_{3} \mathrm{O}_{10}$ crystals were grown directly from the sintered rod at $\mathrm{pO}_{2}=20$ bar using a $3-\mathrm{kW}$ xenon arc lamp to heat the zone. A similar procedure was applied to $\mathrm{Pr}_{4} \mathrm{Ni}_{3} \mathrm{O}_{10}$, but sintering at $1100{ }^{\circ} \mathrm{C}$ was necessary to avoid cracking of the rod. $\mathrm{Pr}_{4} \mathrm{Ni}_{3} \mathrm{O}_{10}$ crystals were grown at $\mathrm{pO}_{2}=$ 140 bar through two steps using a $5-\mathrm{kW}$ xenon arc lamp: a fast pass $(30-50 \mathrm{~mm} / \mathrm{h}$ ) followed by growth at the same pressure at $5 \mathrm{~mm} / \mathrm{h}$. During each growth, a flow rate of $0.1 \mathrm{l} / \mathrm{min}$ of oxygen was maintained. Feed and seed rods were counterrotated at 27 and $20 \mathrm{rpm}$, respectively.

Magnetic susceptibility. Magnetic susceptibility measurements were performed on single crystals using a Quantum Design MPMS3 SQUID magnetometer (The identification of any commercial product or trade name does not imply endorsement or recommendation by the National Institute of Standards and Technology) Specimens were attached to a quartz holder using a minute amount of glue. ZFC-W (Zero-field cooling with data collected on warming), FC-C (field cooling with data collected on cooling) and FC-W (field cooling with data collected on warming) data with magnetic field $\mathbf{H} \| \mathrm{ab}$ were collected between 1.8 and $300 \mathrm{~K}$ under an external field of $0.4 \mathrm{~T}$. The out-of-plane magnetic susceptibility is discussed in a separate paper ${ }^{11}$. In the $\mathrm{ZFC}-\mathrm{W}$ protocol the sample was cooled in zero field to $10 \mathrm{~K}$ at a rate of $35 \mathrm{~K} / \mathrm{min}$ and then to $1.8 \mathrm{~K}$ at a rate of $2 \mathrm{~K} / \mathrm{min}$, and DC magnetization recorded on warming $(2 \mathrm{~K} / \mathrm{min})$. In the $\mathrm{FC}-\mathrm{C}$ and $\mathrm{FC}-\mathrm{W}$ protocols, the magnetization was recorded $(2 \mathrm{~K} / \mathrm{min})$ in a fixed field of $0.4 \mathrm{~T}$.

Electrical resistivity. The resistivity was measured using a four-probe method with contacts made by depositing gold pads. The temperature was controlled using the Quantum Design PPMS in the temperature range of $1.8-300 \mathrm{~K}$.

Heat capacity. Heat capacity measurements were performed on a Quantum Design PPMS (The identification of any commercial product or trade name does not imply endorsement or recommendation by the National Institute of Standards and Technology) in the temperature range of 1.8-300 K. Apiezon-N (The Identification of any commercial product or trade name does not imply endorsement or recommendation by the National Institute of Standards and Technology) vacuum grease was employed to fix crystals to the sapphire sample platform. The specific heat contribution from sample holder platform and grease was determined before mounting sample and subtracted from the total heat capacity.

X-ray diffraction. Synchrotron X-ray single crystal diffraction measurements were performed at beamline 15-ID-D at ChemMatCARS (University of Chicago) at the Advanced Photon Source, Argonne National Laboratory. Data were collected with a $1 \mathrm{M}$ Pilatus area detector using synchrotron radiation $(\lambda=0.41328 \AA)$ at 100 , $110,120,125,127,129,131,133,135,137,139,145,200 \mathrm{~K}$ with temperature controlled by flowing nitrogen gas. To cover a sufficient volume of reciprocal space, $\omega$ and $\varphi$ scans were used, with 1800 frames ( $0.2 \mathrm{deg} /$ frame, continuous scan) collected at each $\omega$ angle. Several single crystals were checked in this experiment, and the SL peaks are reproducible. The 3D reciprocal space volumes were generated from the data using the CCTW package ${ }^{34}$ and visualized using NeXpy ${ }^{35}$, which was used to produce the linecuts. To obtain the precise wavevector, data were collected at beamline $33-B M-C^{36}$ (Advanced Photon Source) using a point detector. The wavevector $\mathbf{q}_{\mathrm{c}}=0.76 \mathbf{b}^{*}$ was obtained by fitting the peaks.

Neutron elastic scattering. Unpolarized measurements in the $h k 0$ and $0 k l$ scattering planes were performed on the MACS cold neutron multi-axis spectrometer at the NIST Center for Neutron Research (NCNR) with $\lambda=4.05 \AA$. Four crystals were coaligned for the $h k 0$, and a crystal of mass $\approx 40 \mathrm{mg}$ was used for $0 \mathrm{kl}$ plane, and data were collected at 1.6 and $180 \mathrm{~K}$. The SDW order parameter was determined by following the evolution of the intensity at $(-1,0.38,0)$ on warming.

Density wave models and simulations. For numerical purposes, the intensities were calculated using 10 unit cells along $c$ (20 trilayers) and $25^{2}$ orthorhombic unit cells in the plane (for a total of $75000 \mathrm{Ni}$ atoms). The slight Bmab deviations of the outer plane $\mathrm{Ni}$ ions from their $14 / \mathrm{mmm}$ positions were taken into account, using atomic coordinates given in ref. ${ }^{11}$ Details are given in the Supplementary Note 6 (Supplementary Figs. 7-11) along with real space stripe simulations in Supplementary Note 7 (Supplementary Fig. 12).

Computational details. We performed first-principles calculations using the WIEN $2 \mathrm{k}^{37}$ code with the Perdew-Burk-Ernzerhof ${ }^{38}$ version of the generalized gradient approximation. A Fourier series spline $\mathrm{fit}^{39}$ to the bands was made with 2736 face centered orthorhombic Fourier functions fit to $448 k$ points in the irreducible wedge of the Brillouin zone. Both the density of states and the susceptibility were calculated using a tetrahedron decomposition of the Brillouin zone ${ }^{40}\left(3 \times 8^{\text {n }}\right.$ tetrahedra in the irreducible wedge with $n=6$ used for the density of states and $n=5$ for the susceptibility). These results are presented in Supplementary Note 9 (Supplementary Fig. 13). 


\section{Data availability}

The data that support the findings of this study are available within the article or from the corresponding author upon reasonable request.

\section{Code availability}

Access to the codes used to calculate density waves and electronic susceptibility will be made available by the corresponding author upon reasonable request.

Received: 24 July 2020; Accepted: 3 November 2020;

Published online: 26 November 2020

\section{References}

1. Catalano, S. et al. Rare-earth nickelates $\mathrm{RNiO}_{3}$ : thin films and heterostructures. Rep. Prog. Phys. 81, 046501 (2018).

2. Zhang, J., Zheng, H., Ren, Y. \& Mitchell, J. F. High-pressure floating-zone growth of perovskite nickelate $\mathrm{LaNiO}_{3}$ single crystals. Cryst. Growth Des. 17, 2730-2735 (2017).

3. Wang, B.-X. et al. Antiferromagnetic defect structure in $\mathrm{LaNiO}_{3-\delta}$ single crystals. Phys. Rev. Mater. 2, 064404 (2018).

4. Dey, K., Hergett, W., Telang, P., Abdel-Hafiez, M. M. \& Klingeler, R. Magnetic properties of high-pressure optical floating-zone grown $\mathrm{LaNiO}_{3}$ single crystals. J. Cryst. Growth 524, 125157 (2019).

5. Zheng, $\mathrm{H}$. et al. Oxygen inhomogeneity and reversibility in single crystal $\mathrm{LaNiO}_{3-\delta}$. Crystals 10, 557 (2020).

6. Li, B. et al. Insulating pockets in metallic $\mathrm{LaNiO}_{3}$. Adv. Electron Mater. 2, 1500261 (2016).

7. Tranquada, J. M. Spins, stripes, and superconductivity in hole-doped cuprates. AIP Conf. Proc. 1550, 114-187 (2013).

8. Yoshizawa, H. et al. Stripe order at low temperatures in $\mathrm{La}_{2-x} \mathrm{Sr}_{x} \mathrm{NiO}_{4}$ with $0.289 \leq x \leq 0.5$. Phys. Rev. B 61, R854-R857 (2000).

9. Zhang, J. et al. Stacked charge stripes in the quasi-2D trilayer nickelate $\mathrm{La}_{4} \mathrm{Ni}_{3} \mathrm{O}_{8}$. Proc. Natl Acad. Sci. USA 113, 8945-8950 (2016).

10. Zhang, J. et al. Spin stripe order in a square planar trilayer nickelate. Phys. Rev. Lett. 122, 247201 (2019).

11. Zhang, J. et al. High oxygen pressure floating zone growth and crystal structure of the metallic nickelates $\mathrm{R}_{4} \mathrm{Ni}_{3} \mathrm{O}_{10}(\mathrm{R}=\mathrm{La}, \mathrm{Pr})$. Phys. Rev. Mater. 4 , 083402 (2020).

12. Huangfu, S. et al. Anisotropic character of the metal-to-metal transition in $\mathrm{Pr}_{4} \mathrm{Ni}_{3} \mathrm{O}_{10}$. Phys. Rev. B 101, 104104 (2020).

13. Kumar, S., Fjellvåg, Ø., Sjåstad, A. O. \& Fjellvåg, H. Physical properties of Ruddlesden-Popper $(\mathrm{n}=3)$ nickelate: $\mathrm{La}_{4} \mathrm{Ni}_{3} \mathrm{O}_{10}$. J. Magn. Magn. Mater. 496, 165915 (2020).

14. Li, B.-Z. et al. Metal-to-metal transition and heavy-electron state in $\mathrm{Nd}_{4} \mathrm{Ni}_{3} \mathrm{O}_{10-\delta}$. Phys. Rev. B 101, 195142 (2020).

15. Huangfu, S., Zhang, X. \& Schilling, A. Correlation between the tolerance factor and phase transition in $\mathrm{A}_{4-x} \mathrm{~B}_{x} \mathrm{Ni}_{3} \mathrm{O}_{10}(\mathrm{~A}$ and $\mathrm{B}=\mathrm{La}, \mathrm{Pr}$, and $\mathrm{Nd} ; x=0,1,2$ and 3). Phys Rev. Research 2, 033247 (2020).

16. $\mathrm{Li}, \mathrm{H}$. et al. Fermiology and electron dynamics of trilayer nickelate $\mathrm{La}_{4} \mathrm{Ni}_{3} \mathrm{O}_{10}$. Nat. Commun. 8, 704 (2017)

17. Seo, D. K., Liang, W., Whangbo, M. H., Zhang, Z. \& Greenblatt, M. Electronic band structure and madelung potential study of the nickelates $\mathrm{La}_{2} \mathrm{NiO}_{4}$ $\mathrm{La}_{3} \mathrm{Ni}_{2} \mathrm{O}_{7}$, and $\mathrm{La}_{4} \mathrm{Ni}_{3} \mathrm{O}_{10}$. lnorg Chem. 35, 6396-6400 (1996).

18. Zhang, Z. \& Greenblatt, M. Synthesis, structure, and properties of $\mathrm{Ln}_{4} \mathrm{Ni}_{3} \mathrm{O}_{10-\delta}$ ( $\mathrm{Ln}=\mathrm{La}, \mathrm{Pr}$, and Nd). J. Solid State Chem. 117, 236-246 (1995).

19. Fawcett, E. Spin-density-wave antiferromagnetism in chromium. Rev. Mod. Phys. 60, 209-283 (1988).

20. Greenblatt, M. Molybdenum oxide bronzes with quasi-low-dimensional properties. Chem. Rev. 88, 31-53 (1988).

21. Zocco, D. A. et al. Pressure dependence of the charge-density-wave and superconducting states in $\mathrm{GdTe}_{3}, \mathrm{TbTe}_{3}$, and DyTe $\mathrm{H}_{3}$. Phys. Rev. B 91, 205114 (2015).

22. Johnston, D. C. Thermodynamics of charge-density waves in quasi onedimensional conductors. Phys. Rev. Lett. 52, 2049-2052 (1984).

23. Wu, G. Q., Neumeier, J. J. \& Hundley, M. F. Magnetic susceptibility, heat capacity, and pressure dependence of the electrical resistivity of $\mathrm{La}_{3} \mathrm{Ni}_{2} \mathrm{O}_{7}$ and $\mathrm{La}_{4} \mathrm{Ni}_{3} \mathrm{O}_{10}$. Phys. Rev. B 63, 245120 (2001).

24. Lee, S. H. \& Cheong, S. W. Melting of quasi-two-dimensional charge stripes in $\mathrm{La}_{5 / 3} \mathrm{Sr}_{1 / 3} \mathrm{NiO}_{4}$. Phys. Rev. Lett. 79, 2514-2517 (1997)

25. Gerber, $\mathrm{S}$. et al. Three-dimensional charge density wave order in $\mathrm{YBa}_{2} \mathrm{Cu}_{3} \mathrm{O}_{6.67}$ at high magnetic fields. Science 350, 949-952 (2015).

26. Soumyanarayanan, A. et al. Quantum phase transition from triangular to stripe charge order in $\mathrm{NbSe}_{2}$. Proc. Natl Acad. Sci. USA 110, 1623-1627 (2013).
27. Ulbrich, H. \& Braden, M. Neutron scattering studies on stripe phases in noncuprate materials. Phys. C. 481, 31-45 (2012)

28. Mukuda, $H$. et al. Uniform mixing of high- $\mathrm{T}_{\mathrm{c}}$ superconductivity and antiferromagnetism on a single $\mathrm{CuO}_{2}$ plane of a $\mathrm{Hg}$-based five-layered cuprate. Phys. Rev. Lett. 96, 087001 (2006).

29. Pynn, R., Press, W., Shapiro, S. M. \& Werner, S. A. Second and third harmonics of the spin density wave in chromium metal. Phys. Rev. B 13 , 295-298 (1976).

30. Morosan, E. et al. Superconductivity in $\mathrm{Cu}_{x} \mathrm{TiSe}_{2}$. Nat. Phys. 2, 544-550 (2006).

31. Kusmartseva, A. F., Sipos, B., Berger, H., Forró, L. \& Tutiš, E. Pressure induced superconductivity in pristine 1T-TiSe ${ }_{2}$. Phys. Rev. Lett. 103, 236401 (2009).

32. Allen, S. J. et al. Gaps and pseudogaps in perovskite rare earth nickelates. $A P L$ Mater. 3, 062503 (2015)

33. Zhang, J. et al. Large orbital polarization in a metallic square-planar nickelate Nat. Phys. 13, 864-869 (2017).

34. Jennings, G. Crystal Coordinate Transformation Workflow (CCTW) (SourceForge). https://sourceforge.net/projects/cctw/ (2019).

35. Osborn, R., Wozniak, J. NeXpy. https://github.com/nexpy/nexpy (2020).

36. Karapetrova, E., Ice, G., Tischler, J., Hong, H. W. \& Zschack, P. Design and performance of the 33-BM beamline at the Advanced Photon Source. Nucl. Instrum. Meth A 649, 52-54 (2011).

37. Blaha, P., Schwarz, K., Madsen, G. K. H., Kvasnicka, D., Luitz J. WIEN2k: An Augmented Plane Wave Plus Local Orbitals Program for Calculating Crystal Properties. (Vienna University of Technology, Austria, 2001).

38. Perdew, J. P., Burke, K. \& Ernzerhof, M. Generalized gradient approximation made simple. Phys. Rev. Lett. 77, 3865-3868 (1996).

39. Koelling, D. D. \& Wood, J. H. On the interpolation of eigenvalues and a resultant integration scheme. J. Comput Phys. 67, 253-262 (1986).

40. Rath, J. \& Freeman, A. J. Generalized magnetic susceptibilities in metals: application of the analytic tetrahedron linear energy method to Sc. Phys. Rev. B 11, 2109-2117 (1975)

\section{Acknowledgements}

This work was supported by the US Department of Energy, Office of Science, Basic Energy Sciences, Materials Science and Engineering Division. The work at Shandong University was supported by the Qilu Young Scholars Program of Shandong University, and the Taishan Scholars Program of Shandong Province. Use of the Advanced Photon Source at Argonne National Laboratory was supported by the U.S. Department of Energy, Office of Science, Office of Basic Energy Sciences, under Contract No. DE-AC0206CH11357. ChemMatCARS Sector 15 is supported by the National Science Foundation under grant number NSF/CHE-1836674. Access to MACS was provided by the Center for High Resolution Neutron Scattering, a partnership between the National Institute of Standards and Technology and the National Science Foundation under Agreement No. DMR-1508249. The authors thank Drs. J. W. Freeland, W. E. Pickett, and Vladimir A Stoica for helpful discussions. J.Z. thanks Dr. Evguenia Karapetrova for her help with the measurements at Beamline 33-BM-C, Advanced Photon Source.

\section{Author contributions}

J.Z., J.F.M., and D.P. conceived and designed the experiments. J.Z. grew single crystals with help from H.Z. J.Z. performed synchrotron X-ray single crystal diffraction with the help of Y.-S.C. and S.G.W. J.Z., S.R., and D.P. performed neutron diffraction with the help of Y.Q. and J.A.R.-R. M.R.N. developed the charge and spin density wave models and performed simulations of the diffraction patterns. A.S.B. and M.R.N. performed DFT calculations and electronic susceptibility calculations. J.Z., D.P., M.K., J.F.M., M.R.N., S.R., and R.O. analyzed data. J.Z., J.F.M., M.R.N., and D.P. wrote the manuscript with contributions from all authors.

\section{Competing interests}

The authors declare no competing interests.

\section{Additional information}

Supplementary information is available for this paper at https://doi.org/10.1038/s41467 020-19836-0.

Correspondence and requests for materials should be addressed to J.Z. or J.F.M.

Peer review information Nature Communications thanks Liu Hao Tjeng and the other, anonymous, reviewer(s) for their contribution to the peer review of this work.

Reprints and permission information is available at http://www.nature.com/reprints

Publisher's note Springer Nature remains neutral with regard to jurisdictional claims in published maps and institutional affiliations. 
(c) (i) Open Access This article is licensed under a Creative Commons Attribution 4.0 International License, which permits use, sharing, adaptation, distribution and reproduction in any medium or format, as long as you give appropriate credit to the original author(s) and the source, provide a link to the Creative Commons license, and indicate if changes were made. The images or other third party material in this article are included in the article's Creative Commons license, unless indicated otherwise in a credit line to the material. If material is not included in the article's Creative Commons license and your intended use is not permitted by statutory regulation or exceeds the permitted use, you will need to obtain permission directly from the copyright holder. To view a copy of this license, visit http://creativecommons.org/ licenses/by/4.0/.

This is a U.S. government work and not under copyright protection in the U.S.; foreign copyright protection may apply 2020 